\title{
Assessment of the Osteogenic Potential of Morphogenetic Protein-2 and Insulin-like Growth Factor-I on Adipose Tissue-Derived Stem Cells
}

\author{
Wael Youssef Elias \\ Faculty of Dentistry, King Abdulaziz \\ University, Jeddah, Saudi Arabia
}

Corresponding author: Wael Youssef Elias

\section{Abstract}

Background Stem cells have been identified as the quintessential cell type for regenerative medicine. This study investigates the effects of bone morphogenic protein-2 (BMP-2) and insulin-like growth factor 1 (IGF-1) on bone differentiation.

Methods and Findings Adipose-derived stem cells (ADSCS) were isolated from fat tissue excised from the inguinal region of a healthy white albino rat. The excised tissues were treated and cultured in special media. Several growth factors were used to differentiate stem cells into osteogenic, adipogenic, neurogenic or chondrogenic lineages, and the effects of BMP-2 and IGF-1 on osteogenic differentiation were assessed. Following isolation and culture, the ADSCs had a spindle-shaped appearance and maintained their mesenchymal-like appearance, indicating an undifferentiated state in a conventional culture medium. The cells began to form colonies from the 10th day of culture and maintained their proliferative capability when cultured in ordinary basic culture medium. When grown in BMP-2 (100 ng/mL) or IGF-1 $(200 \mathrm{ng} / \mathrm{mL})$ alone, ADSCs continued to proliferate. No obvious change in cell morphology was detected under inverted light microscope. Also, no scattered nodules were detected in the field. Alizarin red stain revealed positive effect of combined use of growth factors on ADSCS differentiation.

Conclusion Combined BMP2 and IGF-1 have an osteotropic effect on ADSCs.

Keywords: Cell differentiation; regenerative medicine; adipose derived-stem cells; osteoblasts

Received: September 13, 2015; Accepted: November 13, 2015; Published: November 23, 2015

\section{Introduction}

During skeletogenesis, there are two different manners in which bone formation occurs. Intramembranous or endochondral ossification occur regardless of the embryonic lineage. In both bone formation pathways, osteoblasts play a critical and essential role in bone formation [1,2]. Skeletal tissue is composed of various types of mesenchymal cells (MSCs) such as osteoblasts, chondrocytes, and myoblasts. Bone marrow stromal cells include adipocytes. These cell lineages are believed to originate from common mesenchymal progenitors called pluripotent mesenchymal stem cells [3]. Mesenchymal progenitors acquire tissue specific phenotypes depending on their interaction with various stimulants and their maturation during differentiation. Osteoblasts express various phenotypic markers such as high alkaline phosphatase (ALP) activity. Osteoblastic cells also synthesize collagenous and noncollagenous bone matrix proteins including osteocalcin, osteopontin and bone sialoprotein-2 [4].

Proliferation and differentiation of MSCs into the osteoblastic lineage are regulated by an intrinsic genetically defined program. This program is controlled by various transcription factors, cytokines, morphogens and secreted growth factors. Various hormones and local factors regulate their differentiation such as bone morphogenetic proteins (BMPs) and insulin-like growth factors (IGFs) [5]. 
Adipose tissue derived stem cells (ADSCs) are a growing entity of MSCs that show a very promising future in tissue regeneration both in vivo and in vitro. Adipose tissue derived stem cells are able to differentiate into ectodermal, mesodermal or endodermal lineages, 2 and they can be obtained from either liposuction aspirates or excised fat. One gram of adipose tissue yields approximately 5,000 stem cells [6]. Adipose tissue derived stem cells have been found to be able to keep strong proliferative ability and have a strong multi-differentiation potential [7].

A previous research showed that high-energy electropulsing treatment and high-frequency ultrasonic striking treatment resulted in dramatic microhardness enhancement, and the grain size reached more than twice of the original state [8]. Furthermore, it was shown that improved surface wear resistance and anti-corrosion performance with a strong adherent gradient oxide coating on titanium alloy strips surface could be achieved by highly-efficient electropulsing treatment process, which can be applied in biomedical applications [9].

The aim of the present study was to evaluate the effects of BMP2 and IGF-1 on the proliferation and osteogenic differentiation of stem cells derived from adipose tissue.

\section{Methods}

\section{Sample collection and cell culture}

Adipose tissue derived stem cells were isolated from fat tissue excised from the inguinal region of a healthy white Albino rat. The excised tissues were washed in phosphate buffer saline (PBS). Then the specimens were minced and placed in sterile Eppendorf tubes to which a digesting solution consisting of collagenase type I and dispase mixture (Sigma-Aldrich) was added. Subsequently, the tubes were kept in an incubator for one hour at $37^{\circ} \mathrm{C}$. The cells were then re-suspended at density of 10.000 cells $/ \mathrm{cm}^{2}$ in complete culture medium. The culture medium consisted of

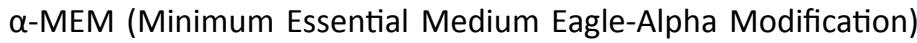
with L-glutamine, (Gibco, Invitrogen Life Technologies, USA), and it was supplemented with $10 \%$ fetal bovine serum (Gibco, Invitrogen Life Technologies, USA), antibiotics (penicillin G, $100 \mathrm{U} / \mathrm{mL}$; streptomycin, $100 \mu \mathrm{g} / \mathrm{mL}$ ) and anti-mycotic agent (Fungizone, $0.25 \mu \mathrm{g} / \mathrm{mL}$ ) in sterile $25-\mathrm{cm}^{2}$, polystyrene, filter cap. Cell-culture plates labeled by cell type and date and incubated in a $\mathrm{CO}_{2}$ incubator at $37^{\circ} \mathrm{C}$ in a humidified atmosphere of $5 \% \mathrm{CO}_{2}$.

\section{Subculturing "passaging"}

The cells were passaged once they became $70 \%$ confluent. Cells were then subcultured every other week. After the third passage, cell cultures were used for cell proliferation and differentiation experiments.

\section{Testing the self-renewal capability of adipose tissue derived stem cells}

To measure the ability of a single cell of ADSCs for colony formation, a suspension of 100 cells $/ \mathrm{mL}$ was cultured in a 3.5 $\mathrm{cm}$ dish in optimal culture medium. Colony formation was then monitored using inverted light microscope. Digital micrographs were taken of the formations.
Cells of the third passage were used for osteogenic differentiation when they reached $70 \%$ confluence by culturing at four different experimental conditions. In all conditions, cells were cultured in $\infty$-MEM fetal bovine serum, containing fetal bovine serum, penicillin-streptomycin and glutamax (ordinary basic culture medium); cells cultured in ordinary basic culture medium only were used as controls. The first group was supplemented with $100 \mu \mathrm{g} / \mathrm{mL}$ BMP-2 (R\&D systems. In the second group, $200 \mu \mathrm{g} / \mathrm{mL}$ IGF-1 (SigmaAldrich) was added. BMP-2 and IGF1 were added together in the third group, both at $200 \mu \mathrm{g} / \mathrm{ml}$. In the fourth group, an osteogenic medium was supplied. This osteogenic medium consisted of L-ascorbic acid (Sigma-Aldrich) $+\beta$-glycerophosphate (Sigma-Aldrich) + dexamethasone (SigmaAldrich).

Daily follow up of the ADSCs growth rate and phenotype change was done using inverted light microscope.

\section{Testing mineralization capability of cultured cells}

Alizarin Red stain was used in a biochemical assay to determine, quantitatively by colorimetry, the presence of calcific deposition inside and around cells of an osteogenic lineage. Bone forming cells containing calcium deposits were stained dark red by the Alizarin Red solution.

Alizarin Red test is considered an early stage marker of matrix mineralization. The cells were rinsed twice with PBS and fixed in $10 \%$ buffered formalin for 10 minutes at room temperature. The fixative was carefully removed and the cells were gently rinsed 3 times with distilled water, followed by staining with $1 \%$ Alizarin Red Solution (Birefringent) for 20 minutes. The Alizarin Red solution was removed and the plates were washed 4 times with 1 $\mathrm{ml}$ distilled water. Aspiration was done after each wash. Finally 1 to $1.5 \mathrm{ml}$ distilled water was added to each plate to prevent cells from drying. The mineralized nodules were stained and monitored using an inverted light microscope and digital micrographs were taken. The results were presented by descriptive analysis.

\section{Evaluation of osteogenic differentiation of cultured cells}

This evaluation was done at two different time intervals of the experiment, on day 12 and on day 22 . This evaluation was done by two methods:

\section{Testing mineralization capability of cultured cells}

Alizarin red stain was used in a biochemical assay to determine, quantitatively by colorimetry, the presence of calcific deposition inside and around cells of an osteogenic lineage. Bone forming cells containing calcium deposits were stained dark red by the Alizarin Red solution.

\section{Reverse transcriptase polymerase chain reaction (RT-PCR)}

The total RNA was extracted from the five groups of cells and RT-PCR was performed to analyze the mRNA level of osteoblastic differentiation markers genes: osteocalcin and bone sialoprotein II. Extraction of RNA was performed using GF-1 total RNA 
Extraction Kit purchased from Vivantis Technologies, USA. The synthesis of complementary DNA (cDNA) was performed using Revert Aid First Strand cDNA Synthesis Kit purchased from Thermo Scientific, USA.

All primers were designed based on target sequences. For the selection of the ideal primer pair, the considered factors included melting temperature $\left(\mathrm{Tm}: 60-65^{\circ} \mathrm{C}\right), \mathrm{GC}$ content $(40 \%-$ $60 \%)$ and amplicon length of about 90-200 bp. Real-time PCR was conducted using Maxima SYBR Green qPCR Master Mix purchased from Thermo Scientific, USA.

\section{Results}

Following the isolation and culturing procedures, the ADSCs had a spindle shaped appearance (Figure 1). Following confluence, the cells were passed successfully up to the third passage. The ADSCs maintained their mesenchymal-like appearance indicating an undifferentiated state in the conventional culture medium (Figure 2)

\section{Colony formation efficiency testing}

Adipose tissue derived stem cells began to form colonies starting from the 10th day of culture.

\section{Testing the proliferation of adipose tissue derived stem cells under different culture conditions}

Adipose tissue derived stem cells maintained their proliferative capability when cultured in the ordinary basic culture medium. This was shown by the increase in cell number and colonies viewed under the inverted light microscope. The cells maintained their original spindle shape.

Adipose tissue derived stem cell groups cultured in both osteogenic media and mixed growth factors changed their morphology and appeared cuboid or ovoid in culture, and they gradually lost their previous spindle-like appearance. Also, scattered nodule-like

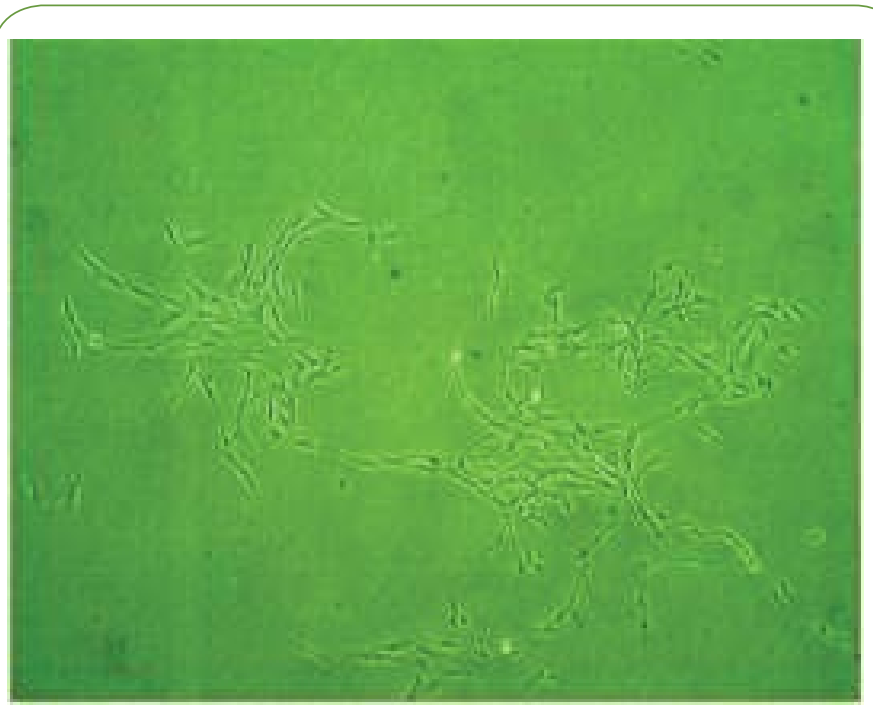

Figure 1 Photomicrograph showing spindle shaped ADSCs isolated at the $10^{\text {th }}$ day of culture (original magnification $X 4$ ).

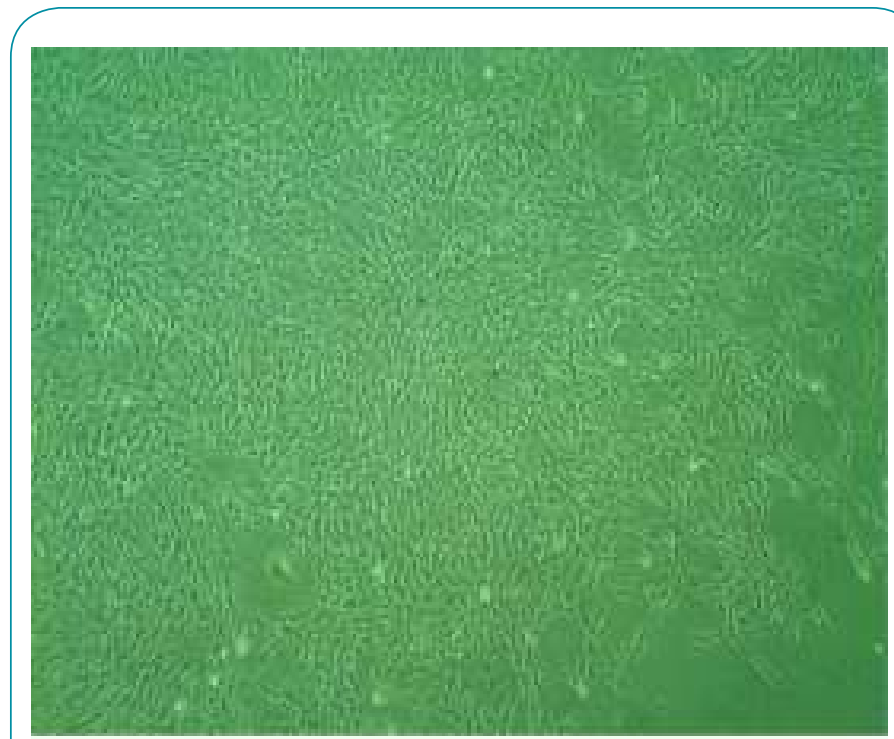

Figure 2 Photomicrograph showing ADSCs reaching 70-80\% confluence. Note the spindle shape of the cells (original magnification $X 4$ ).

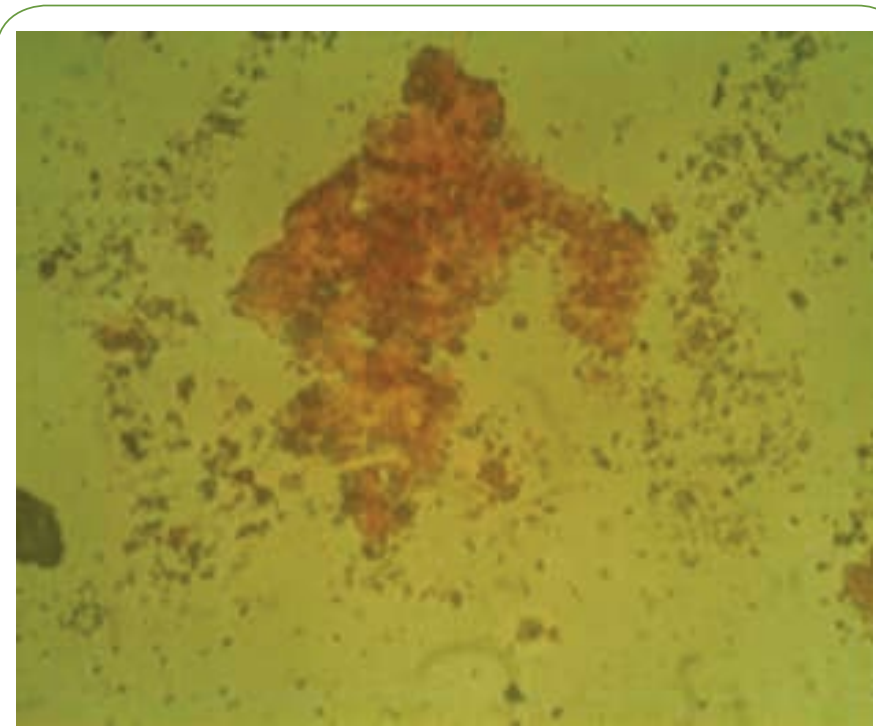

Figure 3 Photomicrograph showing alizarin red stain in osteogenic plates on day 12 indicating beginning of calcification (original magnification $X 4$ ).

structures within the cultured cells started to appear, an event that was not seen in the control plates. Adipose tissue derived stem cells grown in BMP-2 $(100 \mathrm{ng} / \mathrm{mL})$ or IGF-1 $(200 \mathrm{ng} / \mathrm{mL})$ alone continued to proliferate. Meanwhile, no obvious change in cell morphology was detected under inverted light microscope. Also, no scattered nodules were detected in the field.

\section{Alizarin red stain results}

No colorimetric changes were detected in control plates neither on day 12 nor on day 22. Colorimetric changes indicating calcified nodules were detected in osteogenic and mixed growth factors plates both on day 12 and 22. Separate use of BMP-2 and IGF-1 has no effect on calcium ion deposition (Figures 3-6). 
Reverse transcriptase polymerase chain reaction results

Bone sialoprotein-2 and osteocalcin gene were detected in osteogenic plates and mixed growth factors plates on day 12 . On day 22 , the genes were expressed in all plates (Figures 7 and 8 ).

\section{Discussion}

Recently, the role of stem cells for hard tissue formation has considerably increased the attention of researchers. These cells can possibly be a fascinating source of stable differentiated cells, capable of inducing bone formation and control of hydroxyapatite crystal growth [5].

Adipose tissue derived stem cells have several advantages over bone marrow stem cells (BMSCs) from the perspective of clinical application. The yield of cells is much higher from fat than from bone marrow. More so, the proliferation rate of ADSCs are higher than BMSCs [10].

Bone morphogenetic proteins have a key role in bone development and osteoblast differentiation but require interactions with other growth-factor-activated signals. At this juncture, we demonstrate essential crosstalk between BMP-2 and the IGF-1 to initiate osteogenic differentiation in uncommitted mesenchymal precursor cells, and to promote maturation of committed osteoblasts [11].

Dexamethasone, which is the main component of osteogenic media used to enhance healing in bony defects has contrasting dual functions in bone metabolism. Not only is it is a prerequisite supplement for differentiation of stem cells into osteoblasts, it is also a catabolic factor inducing loss of preexisting bone or osteoporosis with prolonged administration in a human system. In addition, dexamethasone is a corticosteroid with all the drawbacks of steroids, most importantly, the delayed healing of surgical sites when applied clinically $[12,13]$. Thus, although an osteogenic medium is the most powerful in osteogenic

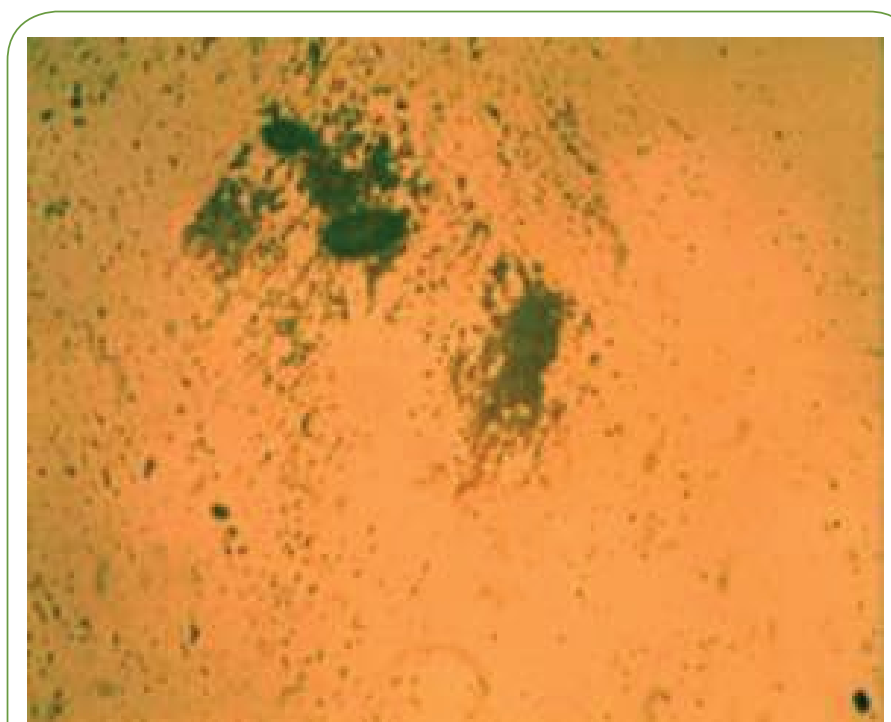

Figure 4 Photomicrograph showing alizarin red stain in growth factors plates on day 12 (original magnification X 4). differentiation of stem cells, our study focused on proving the possibility of substituting the use of an ordinary osteogenic medium containing a steroid by a combination of growth factors (BMP-2 and IGF-1) having a comparative osteogenic capability.

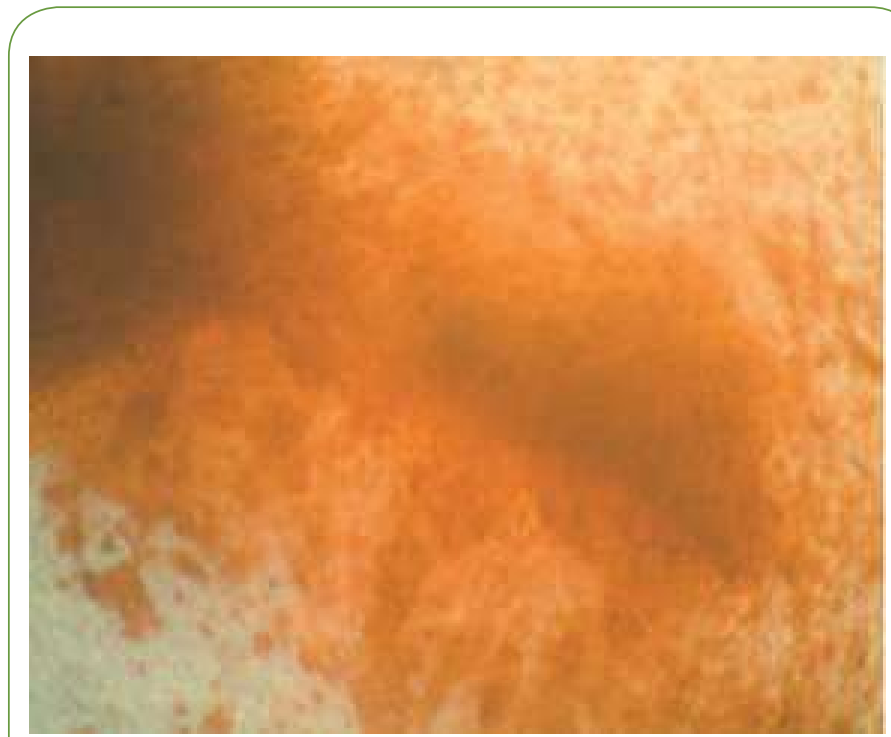

Figure 5 Photomicrograph showing alizarin red stain in osteogenic plates on day 22 (original magnification $\times 20$ ).

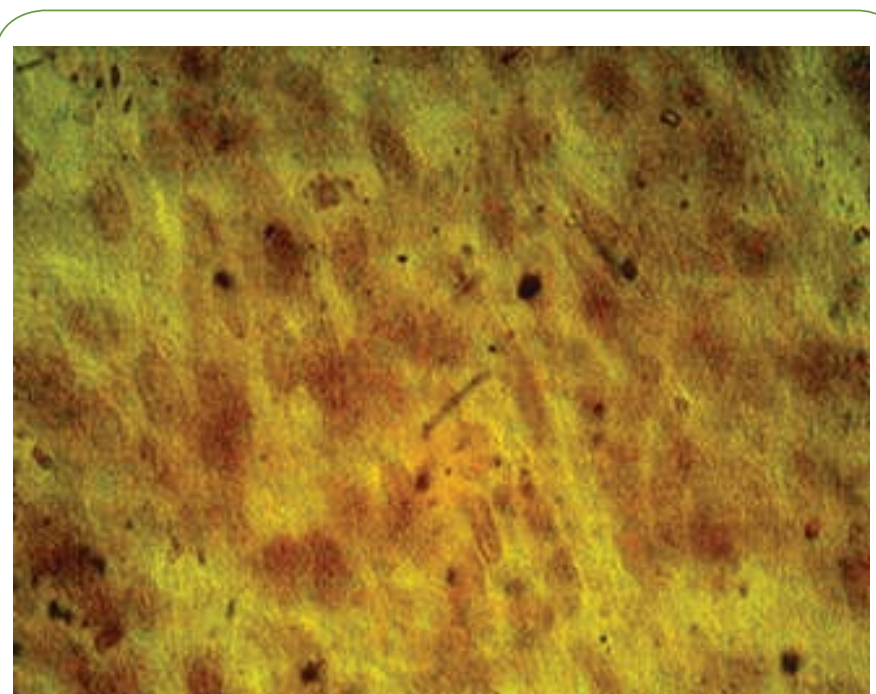

Figure 6 Photomicrograph showing alizarin red stain in growth factors plates on day 22 (original magnification $X 4$ ).

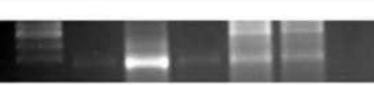

C1 O1 BMP1 CG IGF-I

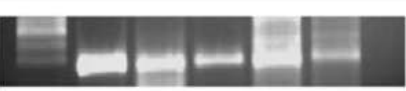

C2 $02 \quad$ BMP2 $\quad$ CG2 IGF-12
Figure 7 A) BSP-2 gene expression on day 12. B) BSP-2 gene expression on day 22. $(\mathrm{C} 1=$ Control plates on day $12 ; \mathrm{C} 2=$ Control plates on day 22; O1= Osteogenic plates on day 12; $02=$ Osteogenic plates on day 22; CG1= Mixed Growth factors on day 12; CG2=Mixed growth factors on day 22). 


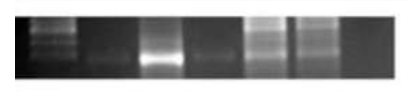

C1 O1 BMP1 CG IGF-I

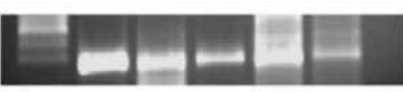

C2 O2 BMP2 CG2 IGF-12
Figure 8 A) Osteocalcin gene expression on day 12. B) Osteocalcin gene expression on day 22. ( $\mathrm{C} 1=$ Control plates on day 12 ; $\mathrm{C} 2=$ Control plates on day 22; 01= Osteogenic plates on day 12; $02=$ Osteogenic plates on day 22; CG1= Mixed Growth factors on day 12; CG2=Mixed growth factors on day 22).

Cells continued to proliferate from the first day until the twenty second day of culture in basic medium, BMP-2 or IGF-1. No change in cell phenotype or in calcium nodule formation was detected, denoting an undifferentiated state under these culture conditions. Cells cultured in osteogenic medium and in combined growth factor medium showed change in cell morphology that may be explained by the start of osteogenic differentiation. This is proven by the formation of calcified nodules. In line with these results, Boland et al. [14] showed that cell proliferation adversely affected the initiation of osteogenic differentiation of adult MSCs.

In the present study, ADSCs expressed bone sialoprotein-2 and osteocalcin genes on the 22nd day of culture in all culture plates, including the control plates, which was not detected from the start of culture. Our results are in accordance with those of De Girolamo et al. [15] who showed that ADSCs cultured in osteogenic media differentiated into osteoblastic cell lineage in vitro and could be induced to form bone tissue in vivo. Patricia et al. [2] showed that expression of the osteoblastic gene, BSP, was upregulated at the ninth passage compared to the first passage. Long term in vitro passage of ADSCs may induce cell differentiation and loss of self renewal ability due to asymmetric cell division.

Taken together, these findings demonstrate that the combination of BMP-2 and IGF-1 can induce osteogenic differentiation of ADSCs. This combination may take a longer time to induce osteogenic differentiation, but it is safer than the osteogenic medium as it is corticosteroid free.

\section{Conclusion}

From the results of the present study it could be concluded that:

1. A combination of BMP-2 $(100 \mathrm{ng} / \mathrm{mL})$ and IGF-I $(200 \mathrm{ng} / \mathrm{mL})$ can be used to substitute the previously cited osteogenic media to induce osteogenic differentiation of ADSCs.

2. This combination may take a longer time to induce osteogenic differentiation with subsequent calcium deposition but it is safer than the osteogenic media as it is corticosteroid free. 


\section{References}

1 Aoki H, Fuji M, Imamura T, Yagi K, Takehara K,et al. (2001)Synergistic effects of different bone morphogenetic protein type I receptors on alkaline phosphatase induction. J Cell Sci 114: 1483-1489.

2 Zuk P, Zhu M, Mizuno H, Huang J, Futrell JW, et al. (2001) Multilineage Cells from Human Adipose Tissue: Implications for Cell-Based Therapies. Tissue Eng 7: 211-228.

3 Kuznetsov S, Krebsbach PH, Satomura K, Kerr J, Riminucci M, et al. (1997) Single-colony derived strains of human marrow stromal fibroblasts from bone after transplantation in vivo. J Bone Miner Res 12: $1335-1347$.

4 Zaidi M (2007) Skeletal remodeling in health and disease. Nat Med 13: $791-801$

5 Yen A, Sharpe P (2008) Stem cells and tooth tissue engineering. Cell Tissue Res 331: 359-372.

6 Strem B, Hicok K, Zhu M (2005) Multipotential differentiation of adipose tissue derived stem cells. J Med 54: 132-141.

7 Zhu Y, Liu T, Song K, Fan X, MA X, et al. (2008) Adipose derived stem cells: a better stem cell than BMSCs. Cell Biochm Funct 26: 664-675.

8 Ye Xiaoxin, Ye Yongda, Tang Guoyi (2014) Effect of electropulsing treatment and ultrasonic striking treatment on the mechanical properties and microstructure of biomedical ti-6Al-4V alloy. J Mech Behav Biomed Mater 40: 287-296.

9 Ye Xiaoxin, Yang Yanyang, Tang Guoyi (2014) Microhardness and corrosion behavior of surface gradient oxide coating on the titanium alloy strips under high energy electro-pulsing treatment. Surface and Coatings Technology 258: 467-484.

10 Breibart A, Grande DA, Kessler R (1998) Tissue-engineered bone repair of calvarial defectsusing cultured periosteal cells. Plast Reconstr Surg 101: 567-574.

11 Li X, Can X (2006) BMP signaling and skeletogenesis. Ann NY Acad Sci 1068: 26-40.

12 Canalis E (1980) Effect of insulin like growth factor I on DNA and protein synthesis in cultured rat calavaria. J Clin Invest 66:709-719.

13 Canalis E, Lian J (1988) Effects of bone associated growth factors on DNA, collagen and osteocalcin synthesis in cultured fetal rat calavariae. Bone 9: 243-246.

14 Boland G, Perkins G, Hall D, Tuan R (2004) Wnt 30 promotes proliferation and suppresses osteogenic differentiation of adult mesenchymal stem cells. J Cell Biochem 93: 1210-1230.

15 De Girolamo L, Sartari MF, Albisetti W, Brini AT. (2007) Osteogenic differentiation of human adipose-derived stem cells: Comparison of two different inductive media. J Tissue Eng Regen Med 1: 154-157. 